\title{
The Association of Functional Polymorphisms of IL-6 Gene Promoter with Stroke: Analysis in a Persian Population
}

\section{Mohammad Reza Ghasemi ${ }^{1}$, Peyman Zargari², Hamdam Hourfar ${ }^{3}$, Maryam Pirhoushiaran ${ }^{1}$, Payam Sasan Nezhad ${ }^{4}$, Mahmoud Reza Azarpazhooh ${ }^{4}$, Ariane Sadr-Nabavi ${ }^{5,1,6^{*}}$}

${ }^{1}$ Department of Medical Genetics, School of Medicine, Mashhad University of Medical Sciences, Mashhad, Iran

${ }^{2}$ Department of Biology, Science and Research Branch, Islamic Azad University, Tehran, Iran

${ }^{3}$ Department of Biology, Faculty of Basic Sciences, Ahar Branch, Islamic Azad University, Ahar, Iran

${ }^{4}$ Department of Neurology, Ghaem Hospital, Mashhad, Iran

${ }^{5}$ Medical Genetic Research Center (MGRC), School of Medicine, Mashhad University of Medical Sciences, Mashhad, Iran

${ }^{6}$ Academic Center for Education, Culture, and Research (ACECR)-Khorasan Razavi, Mashhad, Iran

\section{Article Info:}

\section{A BSTRACT}

Introduction: Sequence variations in genes involved in inflammation system contribute to the risk of several cardiovascular diseases, such as stroke. Interleukin-6, a pleiotropic cytokine, is associated with cardiovascular disease, especially stroke. In other words, cerebral ischemia and inflammation are strongly linked. In the present study, we performed a genetic association study on two single nucleotide polymorphisms (IL6 -572 and -174 G>C) in an Iranian population in Khorasan province. Materials and Methods: Our hypothesis was tested in this case-control study on stroke patients as well as healthy controls that matched for age, sex, and some traditional risk factors. These polymorphisms were detected by Real time Taq man probe and PCR-RFLP. Results: After adjustment for the confounding risk factors, there was an association between the female patients who suffer from stroke and the IL-6 -572 G>C polymorphism and also Hemorrhagic subtype of stroke. Furthermore, there was no association between IL-6 -174 G>C and stroke, exceptone for one allele in male group. Conclusion: In conclusion, genotype of IL-6 -572 G>C May be a genetics risk factor that enhances the risk for stroke in studied population. However, it is highly controversial whether IL-6 -174 G>C is a susceptible gene for stroke in this population. This is needed to be confirmed in a larger population.

Key words:

1. Stroke

2. Inflammation

3. Polymerase Chain Reaction

*Corresponding Author: Ariane Sadr-Nabavi

E-mail:sadrnabavia2009@gmail.com 


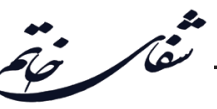

\title{
همبستغى بين يلى مورفيسمهاى عملكردى :بروموتر زن اينترلوكين -9 و سكتهُ مغزى: تجزيه و تحليل در بين يكى جمعيت ايرانى ترونى
}

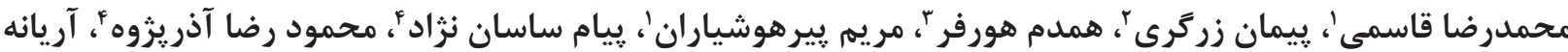

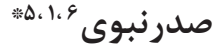

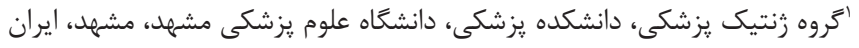

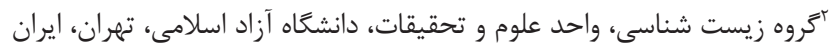

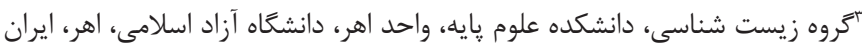

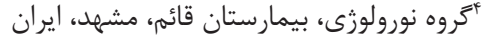

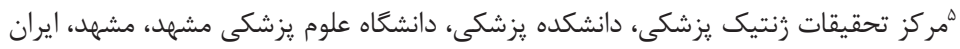

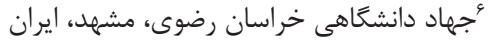

إطلاعات مقاله:

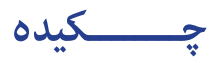

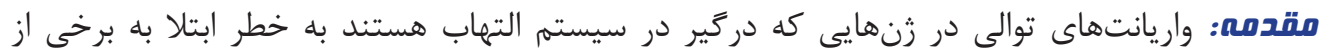

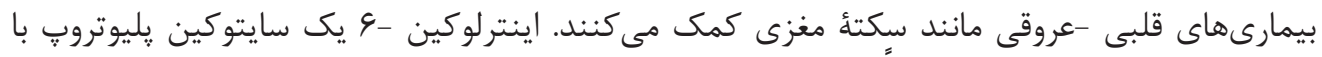

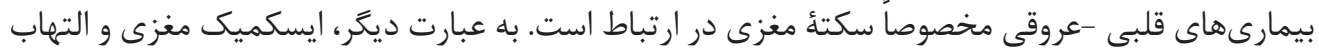

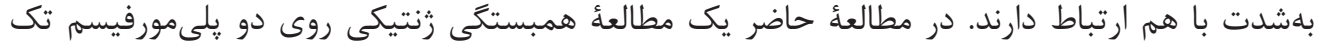

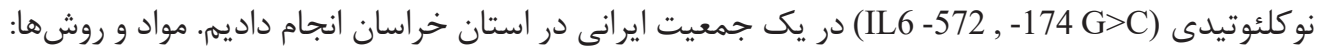

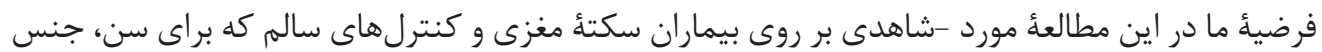

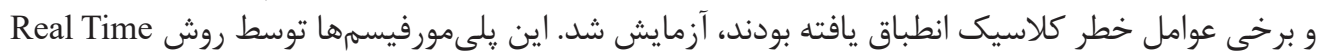
همCR-RFLP و Taq Man Probe

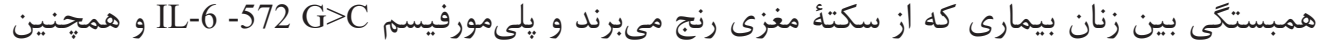

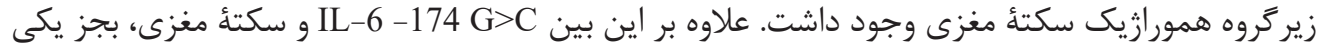

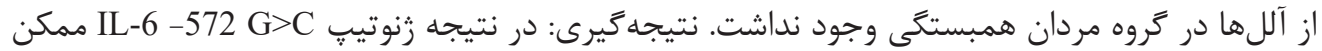

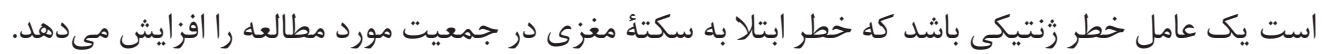

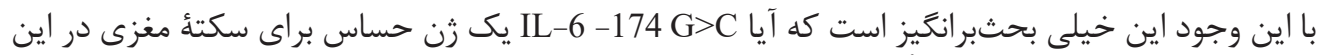

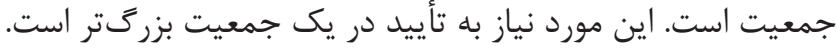

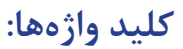

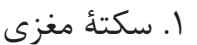
ז. التهاب

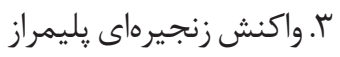

* نويسنده مسئول: آريانه صدرنبوى آدرس الكترونيكى: sadrnabavia2009@gmail.com 


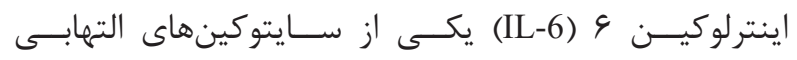

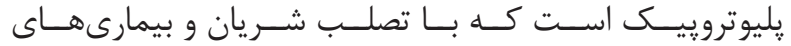

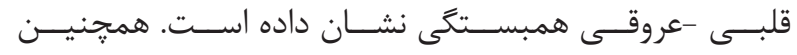

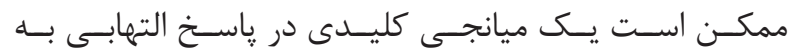

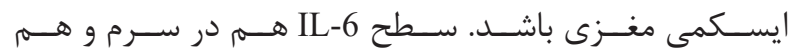

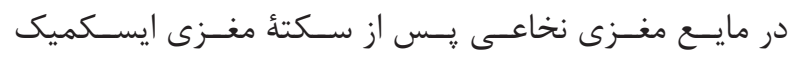

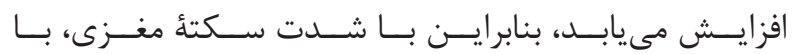

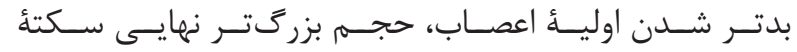

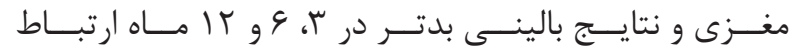

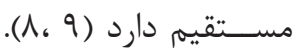

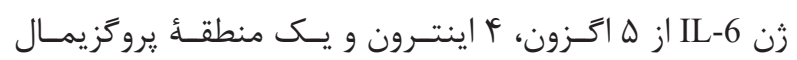

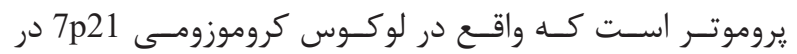

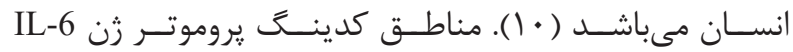

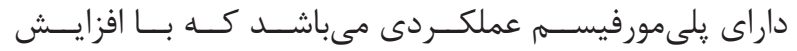

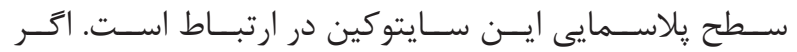

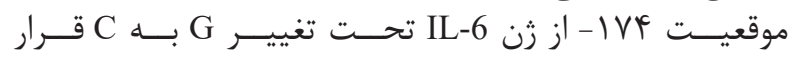

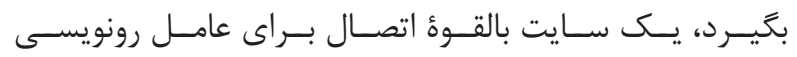

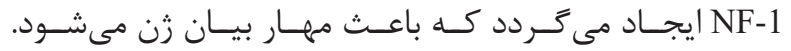

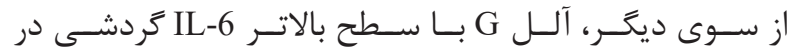

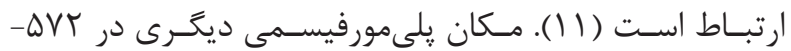

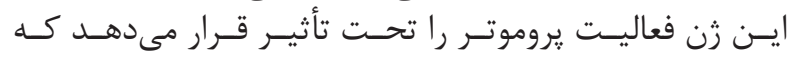

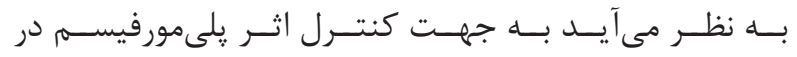

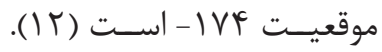

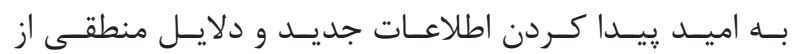

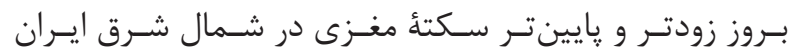

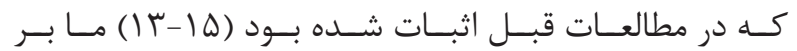

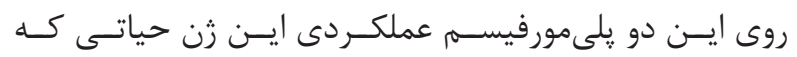

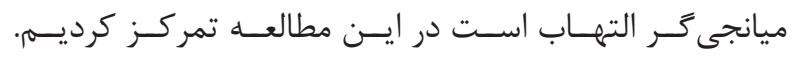

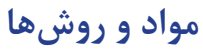

جامعأُ مورد مطالعه

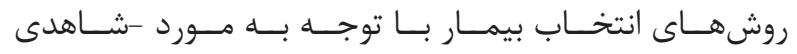

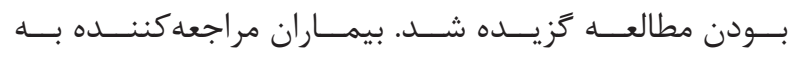

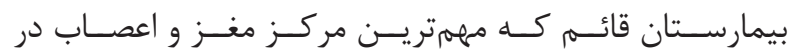

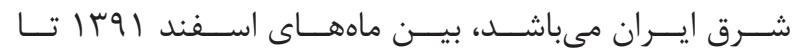

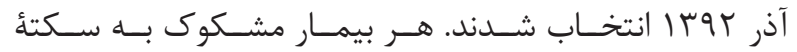

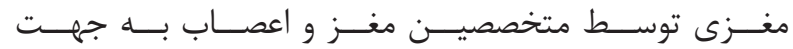

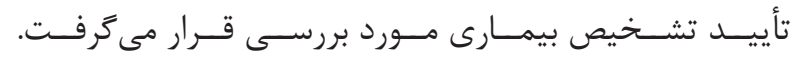

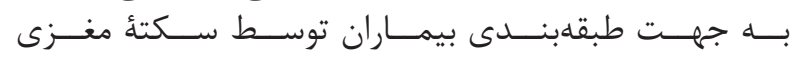

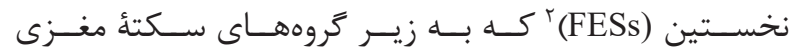

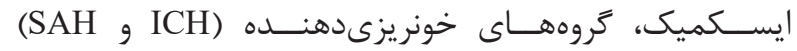

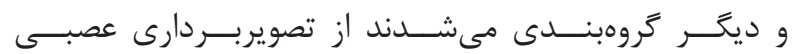

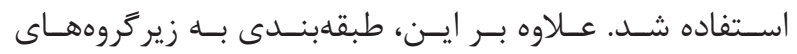

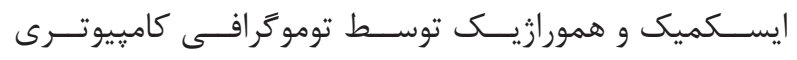

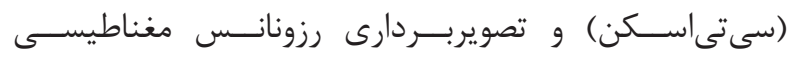

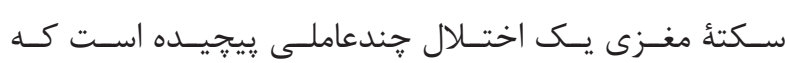

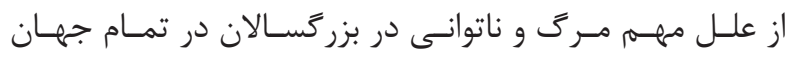

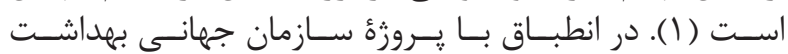

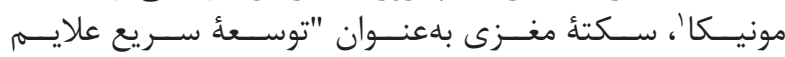

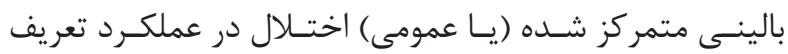

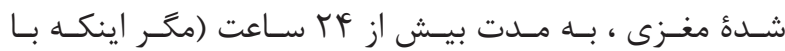

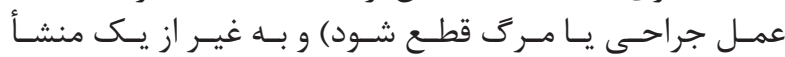

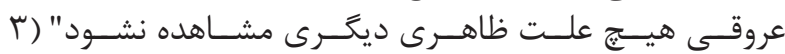

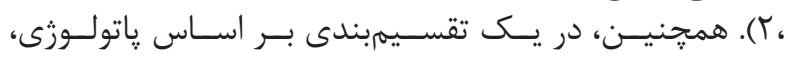

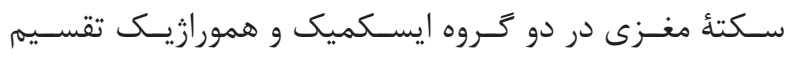

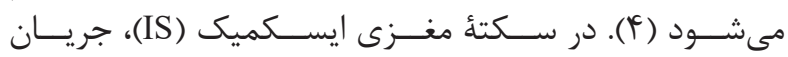

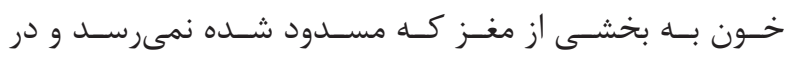

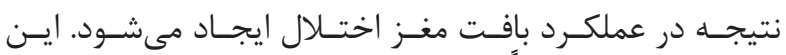

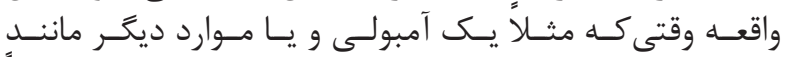

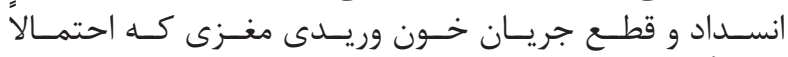

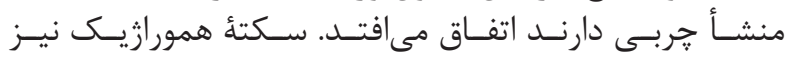

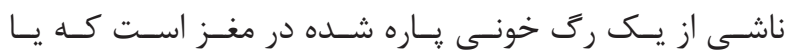

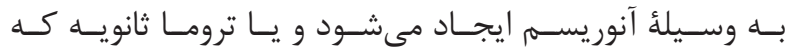

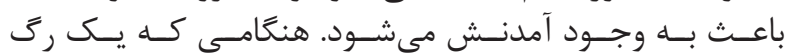

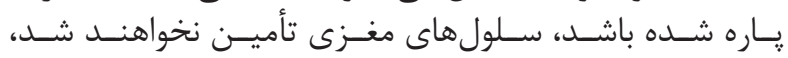

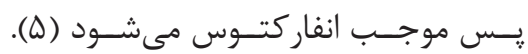

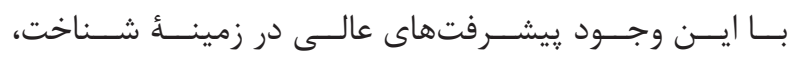

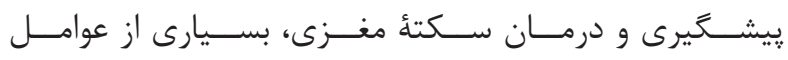

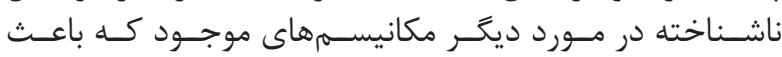

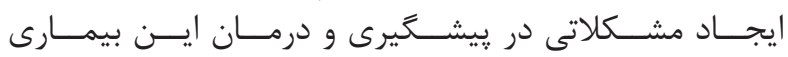

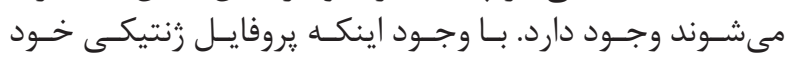

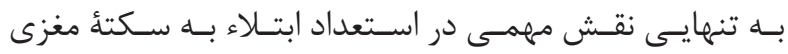

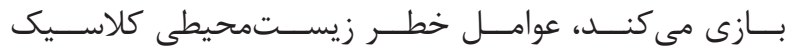

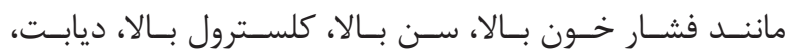

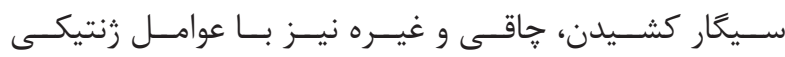

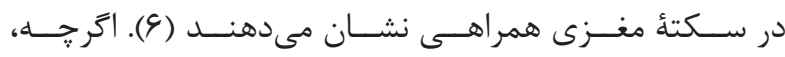

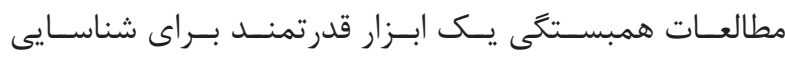

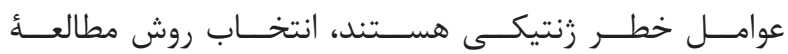

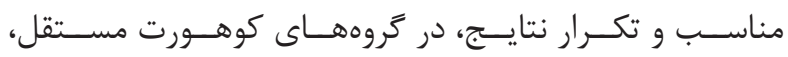

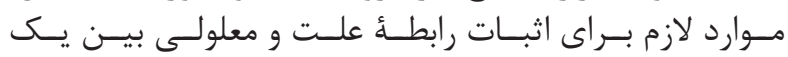

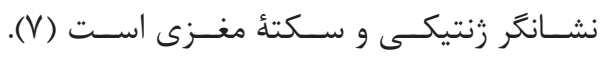

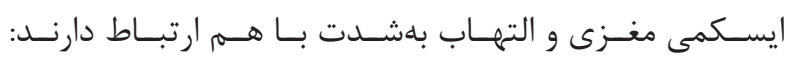

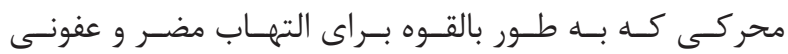

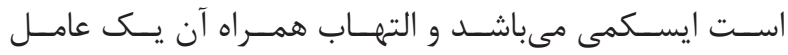

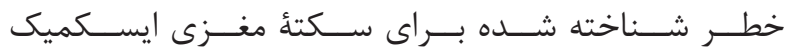

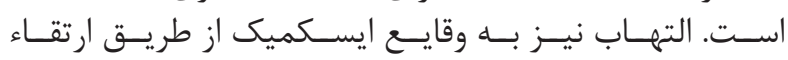

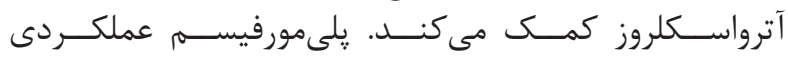

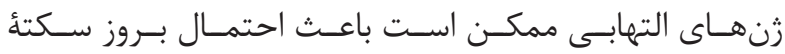

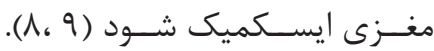


تعيين زُنوتيب

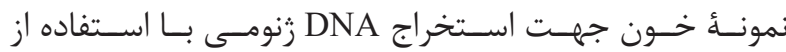

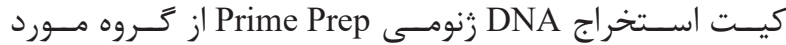

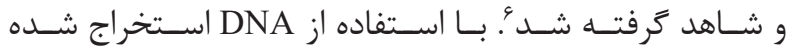

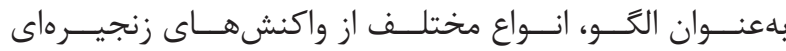

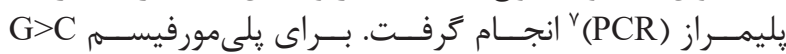

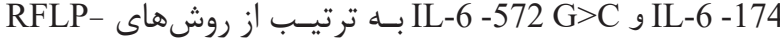
SNP بـ بـراى تعييـن PCR،Real-Time PCR Taqman probe

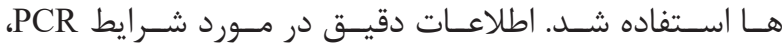

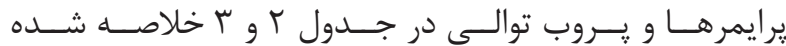

(MRI)

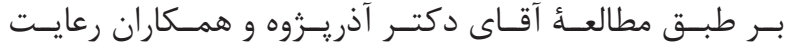

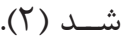

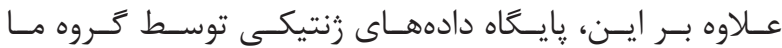

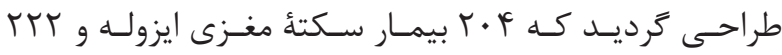

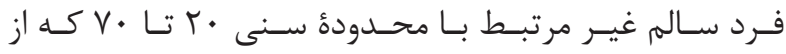

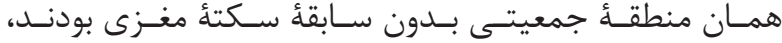

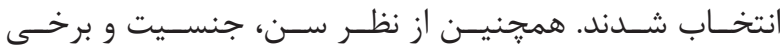

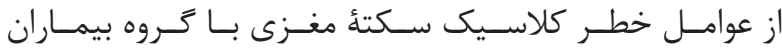

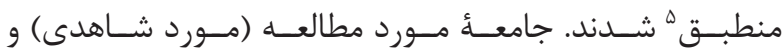

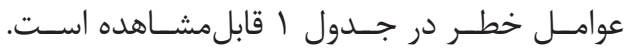

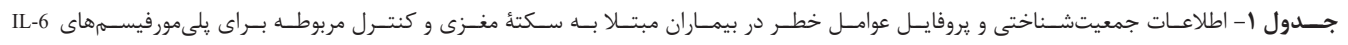

\begin{tabular}{|c|c|c|c|c|c|c|}
\hline \multicolumn{3}{|c|}{ IL-6 -572 G>C } & \multicolumn{3}{|c|}{ IL-6 -174 G>C } & \multirow[b]{2}{*}{ فاكتورهاى خطر مرسوم } \\
\hline تعداد ا+•r & كنترل & ارزش & تعداد r. بيمار & تعداد זنترل & ارزش P & \\
\hline$\Delta \mid / \neg \pm 1 r /$. & $\Delta \cdot / r \pm 11 / V$ &.$/ 119^{*}$ & $\Delta \mid / \wedge F \pm \| r /$. & $\Delta \cdot / \Delta \pm \mid \mathrm{T} / \cdot$ &.$/ T V T^{*}$ & سن (mean \pm SD)* \\
\hline$(11 \Delta / \wedge \varepsilon)$ & $(11 N / \wedge r)$ & ./VArt & $(11 \Delta / \wedge \Lambda)$ & $(11 \Delta / 9 \vee)$ & ./ETrt & جنس (مرد / زن) \\
\hline$\Delta V(Y \wedge)$ & $F \Delta(Y \cdot)$ & $.1 .91 \dagger$ & $\Delta V(Y N / I)$ & $F \Delta(T / / T)$ & $\cdot / 1 \cdot \Delta \dagger$ & ديابت شيرين (./) \\
\hline $1 r V / r \pm r F / F$ & $|r| / \varepsilon \pm r r / r$ &.$/ 110^{*}$ & $|r V /| \pm r F / \Delta$ & $|r| / \Delta \pm r r / \mid$ &.$/ 111^{*}$ & LDL \\
\hline$f \mid \pm q / \Delta$ & $F T / F \pm N / \Lambda$ &.$/ I V r^{*}$ & $f \cdot / q \pm q / \Delta$ & $F \mid / V \pm N / \Delta$ &.$/ r \Delta \Delta^{*}$ & HDL \\
\hline 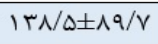 & $\mid f \cdot / f \pm \lambda \cdot / q$ & ./ATV* & $\mid f \cdot / r \pm 9 r / 1$ & $\mid F F / V \pm \Lambda \varepsilon / V$ &.$/ \& T V^{*}$ & Triglyceride \\
\hline $1 \wedge \Delta / \Delta \pm F \Delta / \varepsilon$ & $19 \cdot / \Delta \pm F / / 9$ &.$/$ HAV* $^{*}$ & $1 \wedge \Delta / r \pm F \Delta / q$ & $19 \cdot / 9 \pm r r / r$ &.$/ T \Delta q^{*}$ & Total Cholesterol \\
\hline
\end{tabular}

جدول r- يرايمرها و توالىهاى يروب Taq Man (يرايمر و يروبها بهخصوص، توسط نرمافزار Allele ID طراحى شدهاند).

\begin{tabular}{|c|c|c|}
\hline رن و يلىمورفيسم مربوطه & & توالى هرايمر و يروب ها \\
\hline $\mathrm{IL}-6-174(\mathrm{G}>\mathrm{C})$ & Forward primer & 5'-TTGTCAAGACATGCCAAAGTGCT-'3 \\
\hline $\mathrm{IL}-6-174(\mathrm{G}>\mathrm{C})$ & Reverse primer & 5'-GCCTCAGACATCTCCAGTCC-'3 \\
\hline $\mathrm{IL}-6-572(\mathrm{G}>\mathrm{C})$ & Forward primer & 5'-GCACGAAATTTGAGGATGGC-'3 \\
\hline $\mathrm{IL}-6-572(\mathrm{G}>\mathrm{C})$ & Reverse primer & 5'-TCTGAGTTCTTCTGTGTTCTGG-'3 \\
\hline $\mathrm{IL}-6-572(\mathrm{G}>\mathrm{C})$ & Probe $\mathrm{C}$ & 5-FAM-AGTTCTACAACAGCCCCTCACA-PHOSPHATE3' \\
\hline $\mathrm{IL}-6-572(\mathrm{G}>\mathrm{C})$ & Probe $G$ & 5-HEX-AGTTCTACAACAGCCGCTCACA PHOSPHATE'3 \\
\hline
\end{tabular}

جدول r- شرايط PCR و تعيين زنوتيب.

\begin{tabular}{|c|c|c|c|}
\hline دماهاى مربوط به مراحل PCR & 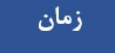 & 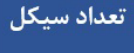 & قطعات آميليكون به جفت باز (bp) \\
\hline $90^{\circ}$ & $\Delta \min$ & ا سيكل & 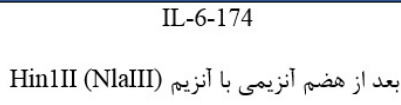 \\
\hline $\begin{array}{c}9 \Delta^{\circ} \\
\left(\text { IL6-174) } \varepsilon \cdot /\left(\text { IL6-572) } \Delta V^{\circ}\right.\right. \\
V Y\end{array}$ & $\begin{array}{l}q \cdot / r \cdot \sec \\
\varepsilon \cdot / r \cdot \sec \\
\varepsilon \cdot / r \cdot \sec \end{array}$ & ه ب سيكل & 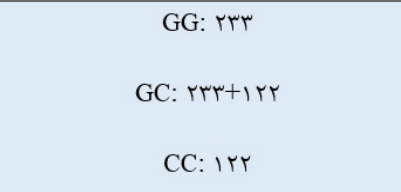 \\
\hline Vr & $\Delta \min$ & 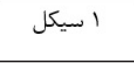 & \\
\hline
\end{tabular}

${ }^{3}$ Magnetic resonance imaging

${ }^{4}$ Case ascertainment

${ }^{5}$ Matched
${ }^{6}$ Catalog No K-2000; genet bio

${ }^{7}$ Polymerase chain reaction 


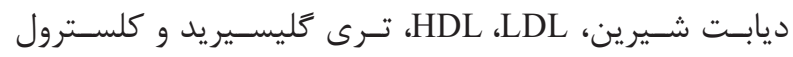

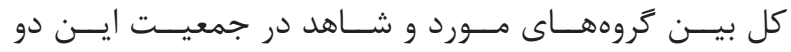

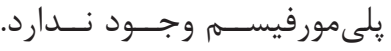
فراوانى زنوتيّ

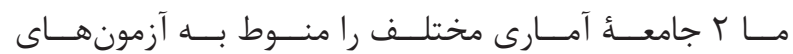

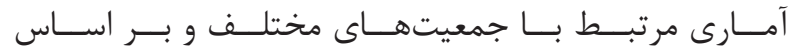

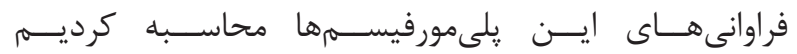

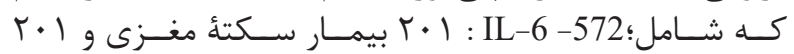

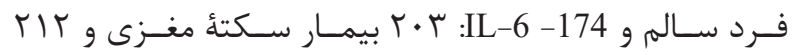

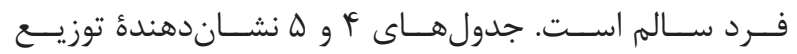

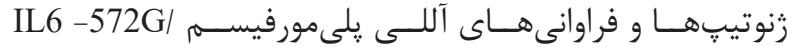

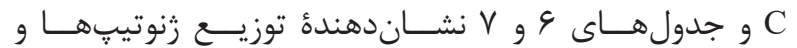

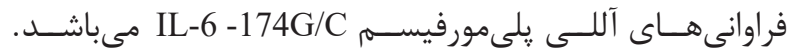

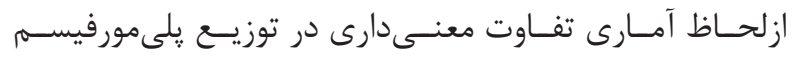

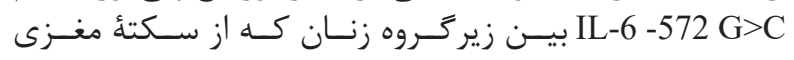

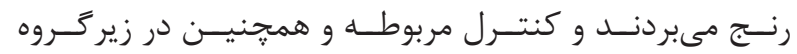

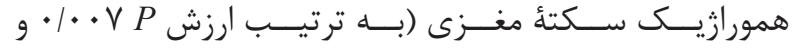

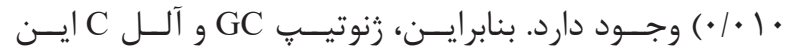

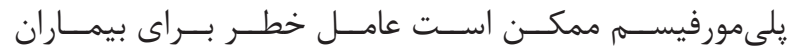

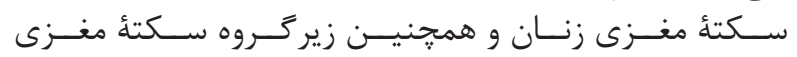

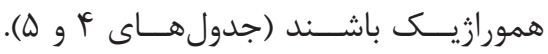

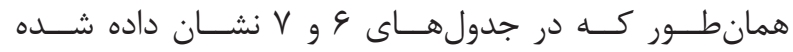

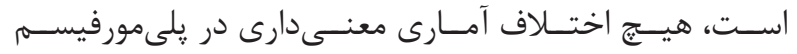

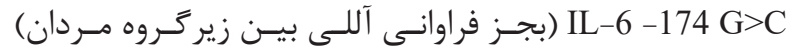

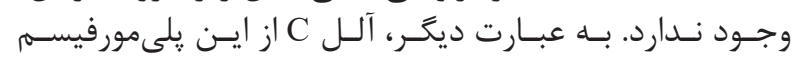

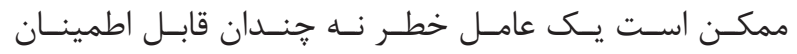

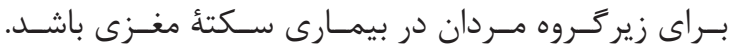

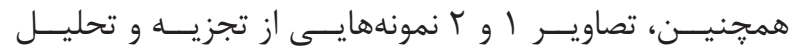

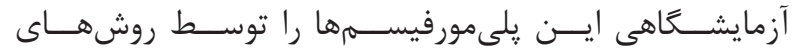

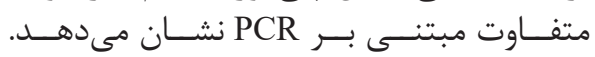

تجزيه و تحليل آمارى

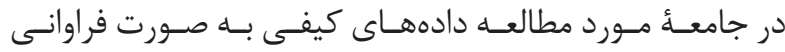

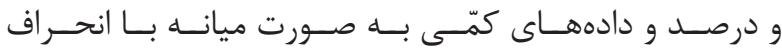

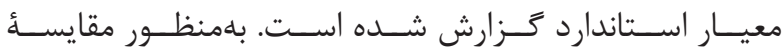

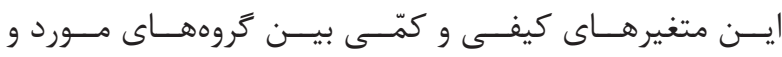

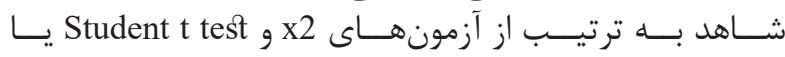

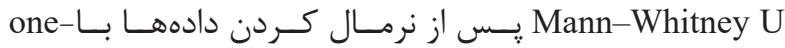
sample Kolmogorov - Smirnov

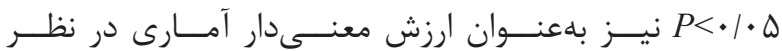

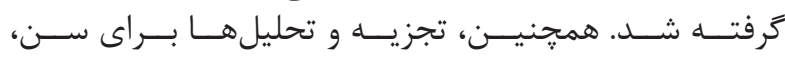

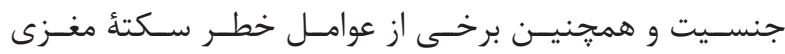

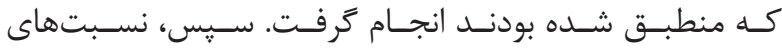

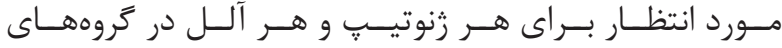

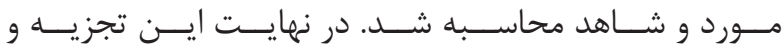

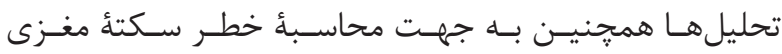

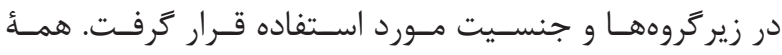

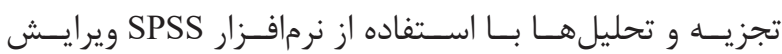

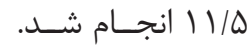

يافتهها

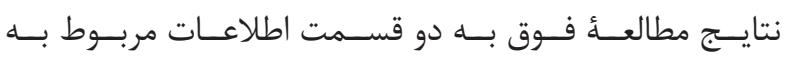

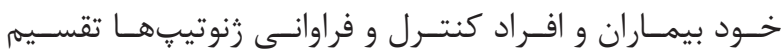

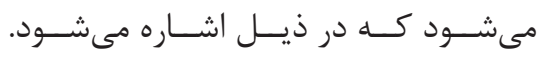
خصوصيات افراد بيمار و كنترل

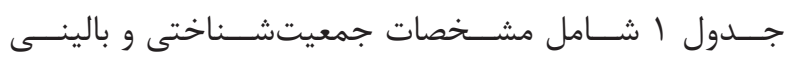

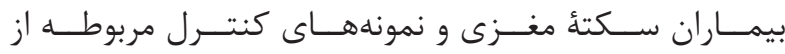

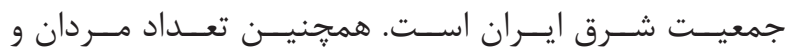

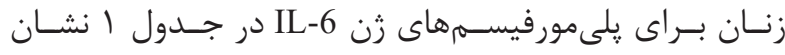

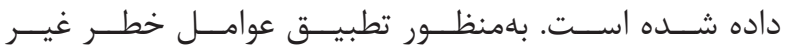

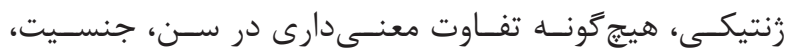

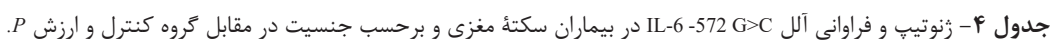

\begin{tabular}{|c|c|c|c|c|c|c|c|c|c|}
\hline $\begin{array}{c}\text { ارزش } \\
\text { P }\end{array}$ & كتداد IV كنت & تعداد هال & $\begin{array}{c}\text { ارزش } \\
P\end{array}$ & كتئرل مرد & تيداد A9 & $\begin{array}{c}\text { ارزش } \\
\text { P }\end{array}$ & تعداد ا•r & تعداد |•بمار & توزيع رُنوتيبى \\
\hline \multirow{3}{*}{$\bullet / . . V$} & $(91 / Q) 1 \cdot V$ & $(V G / Q) \wedge \Lambda$ & \multirow{3}{*}{$\cdot / 111$} & $(A 1 / \cdot) 91$ & $(A V / Y) V \Delta$ & \multirow{3}{*}{$.1 .9 \pi$} & $(A V / I) I V D$ & $(A \mid / I) \mid 9 \Psi$ & (تعدادو \%) GG \\
\hline & . & $(. / 9) 1$ & & . & $(r / T) Y$ & & . & $(1 / Q)^{r}$ & (تعداد و (\%) CC \\
\hline & $(A / Q) V \Psi$ & $(T Y / Y) Y G$ & & $(19 / \cdot) 19$ & $(1+/ Q) 9$ & & $(I K / q) Y \xi$ & $(I V / \mathcal{F}) \Gamma \Delta$ & (تعدادو و) GC \\
\hline $\begin{array}{c}\text { ارزش } \\
\text { P }\end{array}$ & تعنترل زن & تعداد· بيمار زن & $\begin{array}{c}\text { ارزش } \\
\text { P }\end{array}$ & 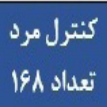 & 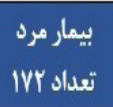 & $\begin{array}{c}\text { ارزش } \\
\text { P }\end{array}$ & تعداد Y Fثترل & 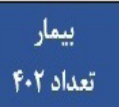 & توزيع آللى \\
\hline \multirow{2}{*}{.. $.4 r$} & $(q \Delta / \mathrm{V}) \mathrm{KYF}$ & $(\Lambda V / \Lambda) r \cdot r$ & \multirow{2}{*}{.$/ A 19$} & $(q \cdot / Q) \wedge T$ & $(9 \% / F) 109$ & \multirow{2}{*}{$.1 . \Delta 9$} & $(q F / Q) r v G$ & $(\Lambda 9 / 9) \% 91$ & (تعدادو و\%) G \\
\hline & $(\boldsymbol{F} / \Psi) 1$. & (IT/Y) YA & & $(9 / A) 19$ & (V/9) IT & & $(G / D) Y G$ & $(1 \cdot / r) \mathrm{rI}$ & C C (تعداد و C C \\
\hline
\end{tabular}


حدول ه- ثنوتيب و فراوانى آلل IL-6 -572 G>C در بيماران سكتأ مغزى و برحسب زيركروه در مقابل گروه كنترل و ارزش P.

\begin{tabular}{|c|c|c|c|c|c|c|c|c|c|}
\hline $\begin{array}{c}\text { ارزش } \\
\text { P }\end{array}$ & تعداد ا+r r كثرل & تعداد بئر بيماران & ارزش & تعداد ا+r & تعدورائيك تعداد & $\begin{array}{c}\text { ارزش } \\
\mathbf{P}\end{array}$ & تعداد ا+بr & تعداد ايسميكى & توزيع رثوتيبى \\
\hline \multirow{3}{*}{. $\mid 911$} & $(91 / \Delta) 1+V$ & $(A T / r) T$. & \multirow{3}{*}{.1 .1} & $(\Lambda) / *) 9 \Lambda$ & $(V \Delta / \Lambda) \Delta^{*}$ & \multirow{3}{*}{. /rrf } & $(A \vee / 1) I V \Delta$ & $(\Lambda r / \Lambda)$ qr & (تعداد و \%G GG \\
\hline & - & - & & . & $(r / \cdot) r$ & & * & (1) 1 & CC (تعداد و \%) CC \\
\hline & $(\Lambda / \Delta) \mathrm{Vr}$ & $(19 / V) F$ & & $(19 / \cdot) 19$ & $(T) / T) 1 f$ & & $(1 T / 9) T Q$ & $(1 \mathrm{Q} / \mathrm{T}) \mid \mathrm{V}$ & (تعداد و \% GC \\
\hline $\begin{array}{c}\text { ارزش } \\
\text { P }\end{array}$ & تعداد Yثترل & تعداه PA بيماران & $\begin{array}{c}\text { ارزش } \\
\mathbf{P}\end{array}$ & 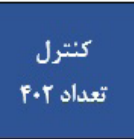 & تعمورائيك & $\begin{array}{c}\text { ارزش } \\
\mathbf{P}\end{array}$ & تعداه r.r & تعداد بيميك & توزيع آللى \\
\hline \multirow{2}{*}{. laTf } & $(a r / \Delta) r v g$ & (9)/V) $\mathrm{FF}$ & \multirow{2}{*}{.1 .09} & $(a r / D)$ rvg & $(\Lambda q / F) \| f$ & \multirow{2}{*}{. Irrf } & $(q r / \Delta) r v g$ & (9)/F) r.r & (تعداد و \%) G \\
\hline & $(g / \Delta) T G$ & $(\Lambda / T) F$ & & $(g / \Delta) T \varphi$ & $(1 \mathrm{r} / 9) \mid \mathrm{A}$ & & $(g / \Delta) T G$ & $(1 / 9) 19$ & (تعداد و \%) C \\
\hline
\end{tabular}

جدول 9- رنوتيب و فراوانى آلل IL-6 -174 G>C در بيماران سكتهٔ مغزى و برحسب جنسيت در مقابل كروه كنترل و ارزش P.

\begin{tabular}{|c|c|c|c|c|c|c|c|c|c|}
\hline $\begin{array}{c}\text { ارزش } \\
\text { P }\end{array}$ & 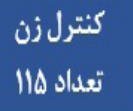 & تعداد ه|| بيمار زن & $\begin{array}{c}\text { ارزش } \\
\text { P }\end{array}$ & كتدرل مرد & تعداد 19 & ارزش & كنداد كוا & تعداد ب. ب ب & توزيع رُنوتيبى \\
\hline \multirow{3}{*}{.1099} & (99/1) $\mathrm{rr}$ & $(9 \Delta / T) \vee \Delta$ & \multirow{3}{*}{$\cdot \mid \cdot \Delta F$} & $(1 \Delta / \Delta) 1 \Delta$ & (Yq/Y) YG & \multirow{3}{*}{.94.} & ו"וז/A) & $(9 / / 9) \| \pi \Delta$ & (تعداد و GG GG \\
\hline & $(Y / 9) \Gamma$ & $(. / 9) 1$ & & $(r q / Q) r q$ & $(T \cdot / r) 1 \mathrm{~A}$ & & $\left(Y / F^{*}\right) \Delta$ & $(\Psi / *) 9$ & (تعداد و CC CC \\
\hline & (ז// & q & & $(\Delta F / \varphi) \Delta \Psi$ & $(\omega \cdot 19) \& \Delta$ & & $(\Gamma \Delta / \Lambda) \vee G$ & $(T \Delta / \Delta) V Y$ & (تعداد و GC GC \\
\hline $\begin{array}{c}\text { ارزش } \\
\text { P }\end{array}$ & تعدترل زن & تعداد • بران & $\begin{array}{c}\text { ارزش } \\
\text { P }\end{array}$ & 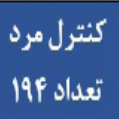 & $\begin{array}{l}\text { تيمار مرد } \\
\text { IVA }\end{array}$ & ارزش P & تعداد كتترل & تعداد P.9. P & توزيع آللى \\
\hline \multirow{2}{*}{.9 .4} & $(A) / V) 1 A \Lambda$ & $(A Y / Y) 1 A 9$ & \multirow{2}{*}{$.1 . \mathrm{YF}$} & $(\mathcal{E r} / \Lambda) \wedge \mu$ & $(\Delta F / Q) q Y$ & \multirow{2}{*}{$\cdot \mid \Lambda \wedge \Delta$} & (VQ/V) & $(V Q / T) H Y r$ & (تعداد و \%) G \\
\hline & $(1 \mathrm{~N} / \mathrm{T} / \mathrm{Fr}$ & $(I V / A) F \mid$ & & $(\Delta V / T) \| I I$ & $(F \Delta / \Delta) \wedge I$ & & $(Y+/ r) \wedge 9$ & $(r \cdot / V) \wedge F$ & (تعداد و \%) C \\
\hline
\end{tabular}

جدول V- رنوتيب و فراوانى آلل IL-6 -174 G>C در بيماران سكته مغزى و برحسب زيركروه در مقابل گروه كنترل و ارزش P.

\begin{tabular}{|c|c|c|c|c|c|c|c|c|c|}
\hline ارزش & كثترل & 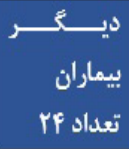 & $\begin{array}{c}\text { ارزش } \\
\mathbf{P}\end{array}$ & كتيترل & تعموراريك بيمار & $\begin{array}{c}\text { ارزش } \\
\text { P }\end{array}$ & كتبرل & تعداد & توزيع رنوتييى \\
\hline \multirow{3}{*}{$\cdot / \Delta \cdot \Lambda$} & | & Tו & \multirow{3}{*}{. ITVG } & וזו (G)/A) & $(V / / Y) F V$ & \multirow{3}{*}{$\cdot|\Delta| \mid$} & וTI/A) & $(\Delta V / \Delta) 9 \Delta$ & (تعداد و .\% GG \\
\hline & $(Y / F) \Delta$ & . & & $(Y / F) \Delta$ & $(1 / \Delta) 1$ & & $(Y / F) \Delta$ & $(F / F) \Delta$ & (تعداد و CC \\
\hline & $(r \Delta / \Lambda) \vee G$ & $(F \Delta / A) \|$ & & $(r \Delta / \Lambda) V G$ & $(Y V / T) \mid \Lambda$ & & $(T \Delta / \Lambda) \vee G$ & $(\mathrm{rN} / \mathrm{N}) \mathrm{Fr}$ & (تعداد و GC \\
\hline $\begin{array}{c}\text { ارزش } \\
\text { P }\end{array}$ & تعداد FHF & تيماران & $\begin{array}{c}\text { ارز } \\
\mathbf{P}\end{array}$ & كثبترل & تعموراريك تيمار & $\begin{array}{c}\text { ارزش } \\
\text { P }\end{array}$ & كنترل & تيمار & توزيع آللى \\
\hline \multirow{2}{*}{.1999} & (Vq/V) rrA & $(V V / I) r V$ & \multirow{2}{*}{.$/ 19}$. & ג & $(\Lambda F / \Lambda) \| r$ & \multirow{2}{*}{.$/ T F A$} & $(V q / V) r r \Lambda$ & $(\Delta Y / Y) I V T$ & (تعداد و \%) G \\
\hline & $(r \cdot / r) \wedge \xi$ & $(Y r / 9) 11$ & & $(T \cdot / T) \wedge 9$ & $(I Q / Y) Y$. & & $(T \cdot / T) \wedge 9$ & (Tr/Q & C (تعداد و \%) \\
\hline
\end{tabular}




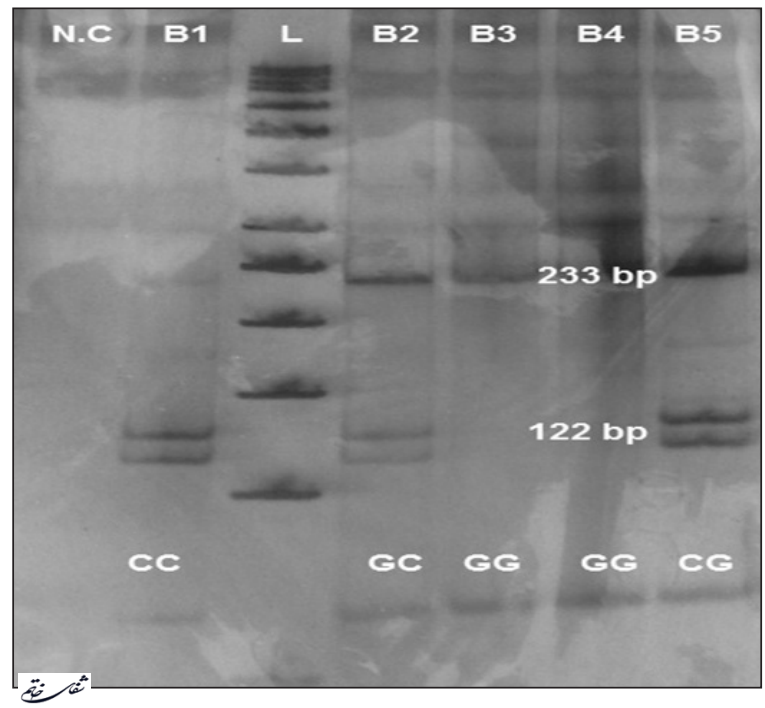

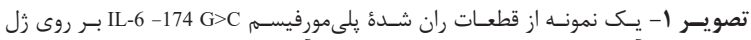

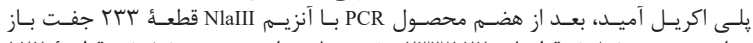

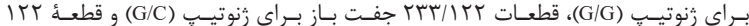

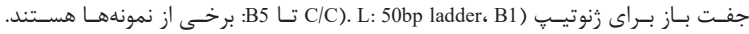

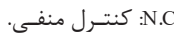

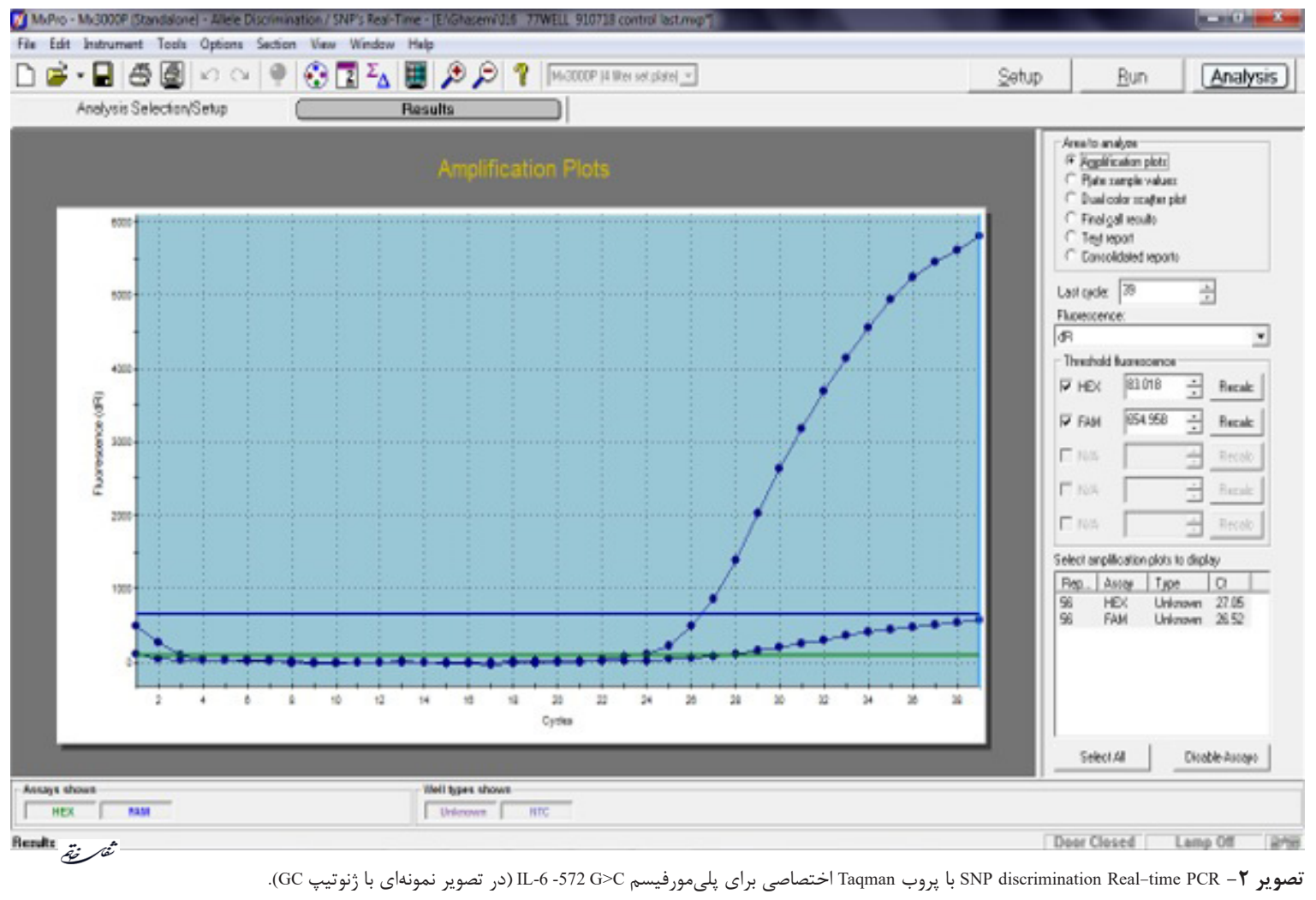

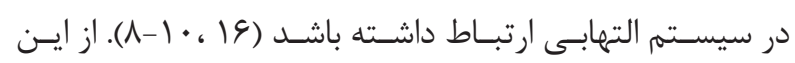

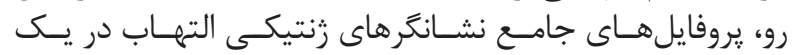

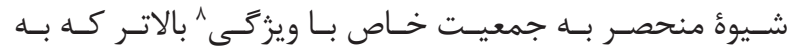

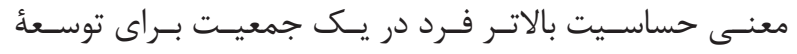

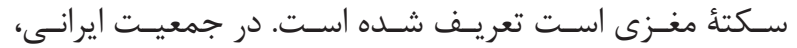

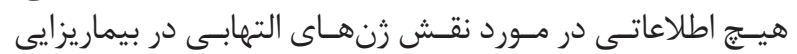

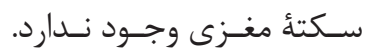

بحث و نتيجه

على غـــم نتايــج نامطلــوب در مــورد سـختى تعييـن ارتبــاط

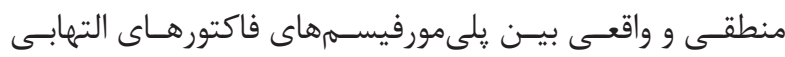

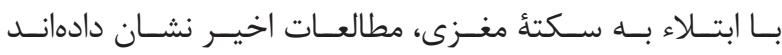

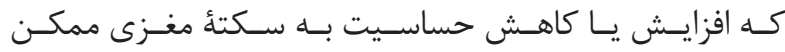

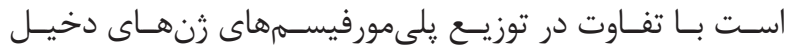

\footnotetext{
${ }^{8}$ Specificity
} 


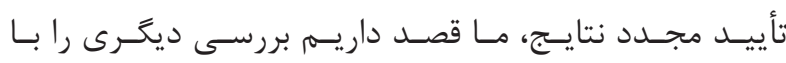

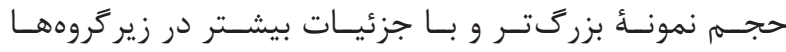

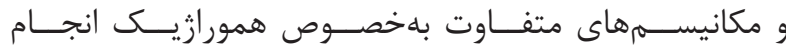

دهيـم.

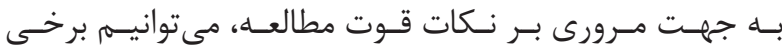

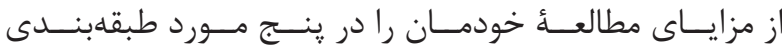

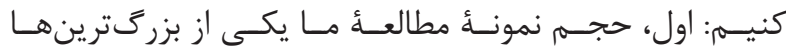

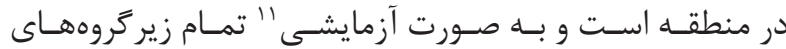

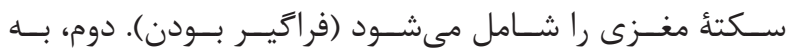

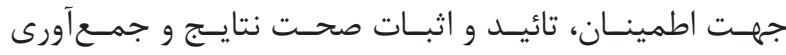

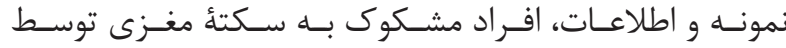

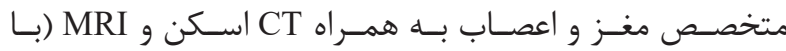

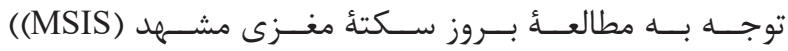

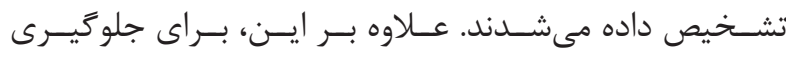

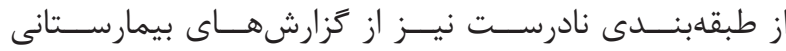

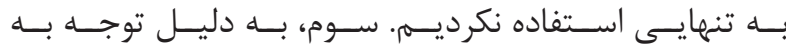

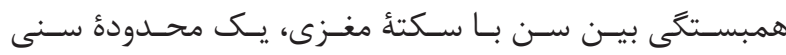

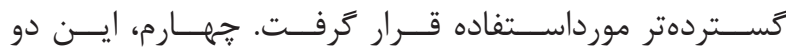

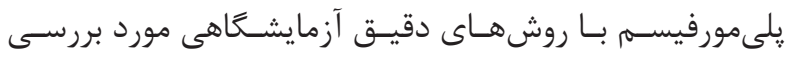

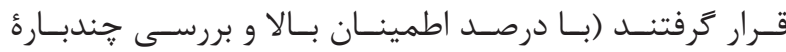

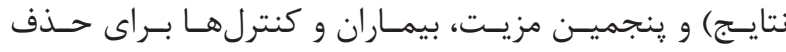

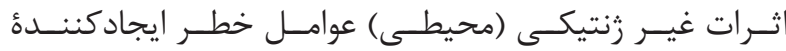

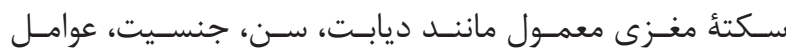

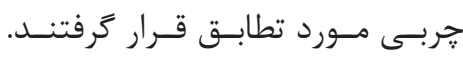

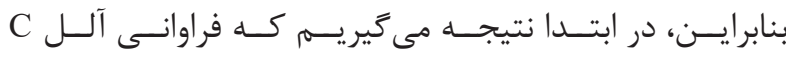

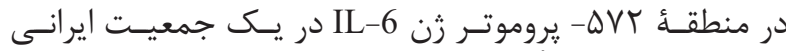

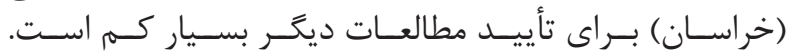

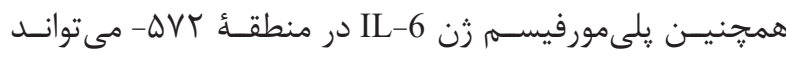

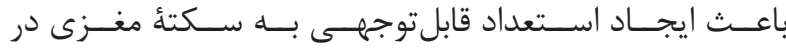

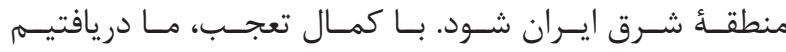

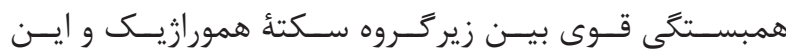

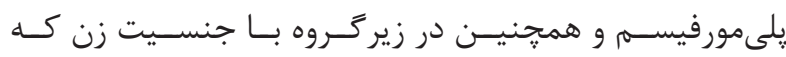

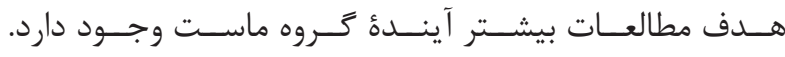

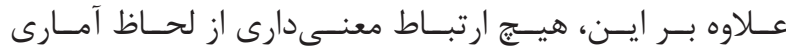

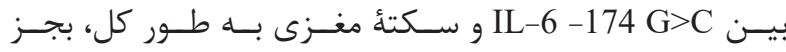

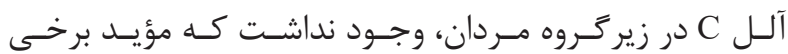

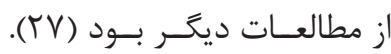

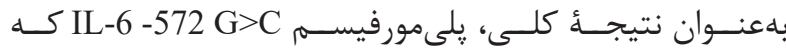

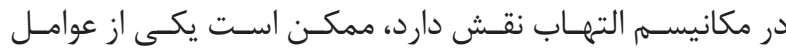

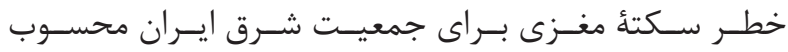

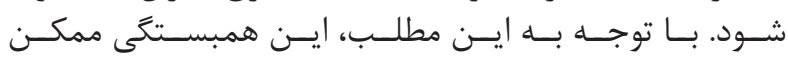

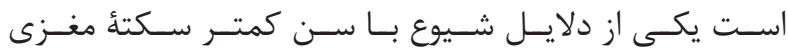

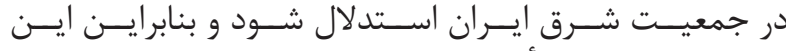

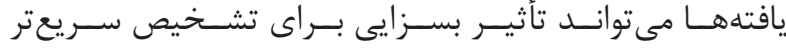

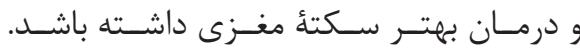

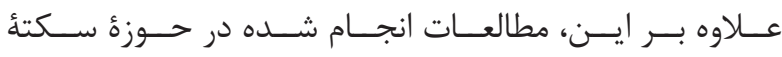

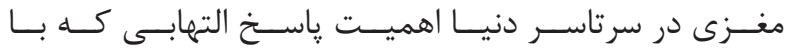

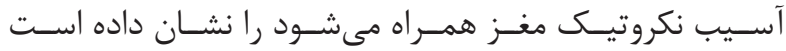

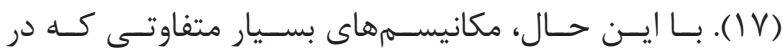

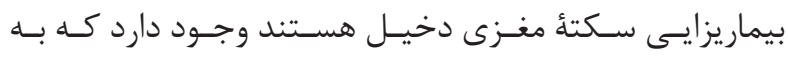

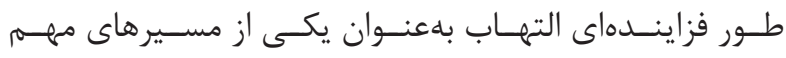

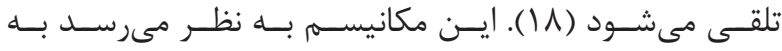

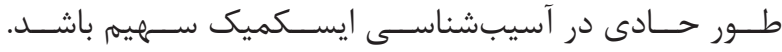

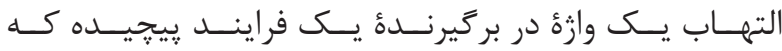

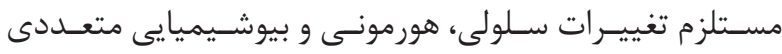

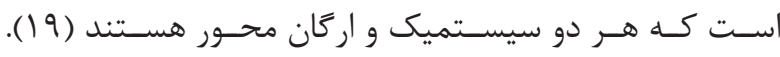

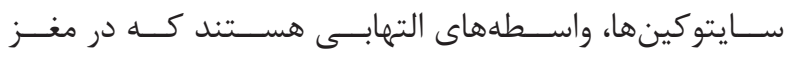

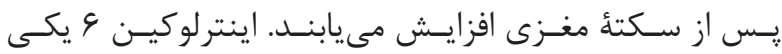

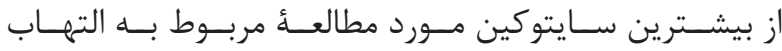

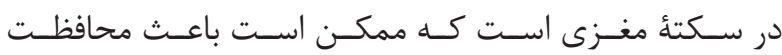

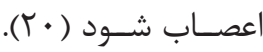

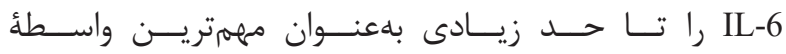

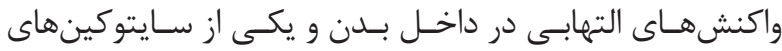

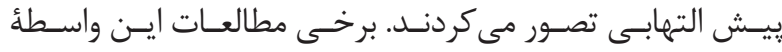

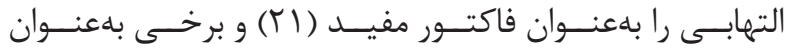

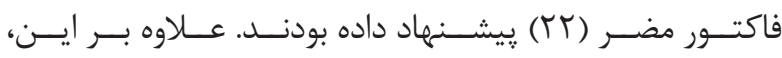

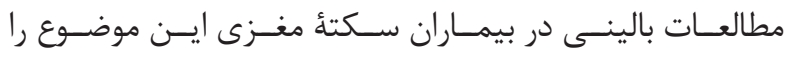

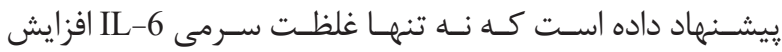

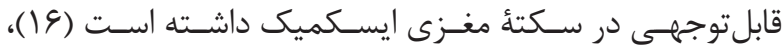

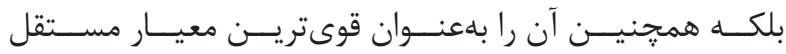

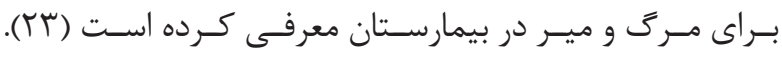

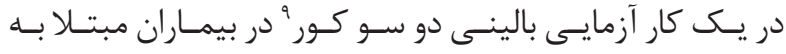

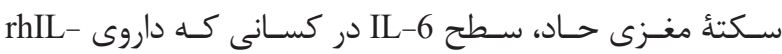

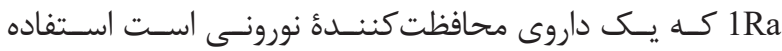

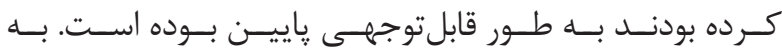

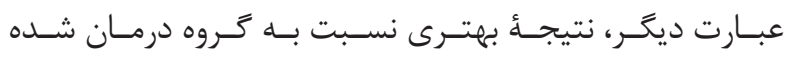

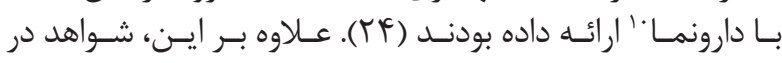

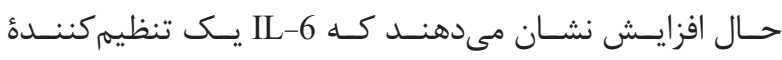

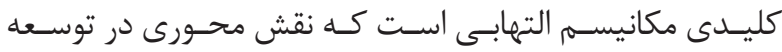

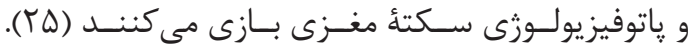

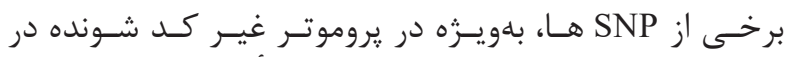

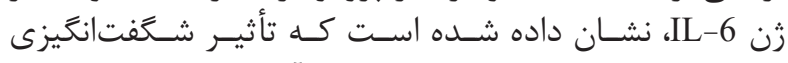

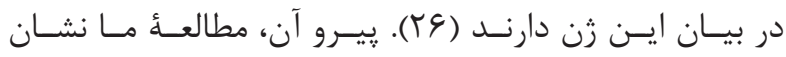

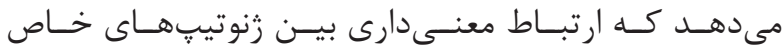

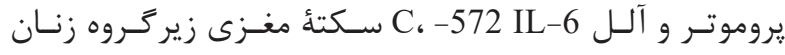

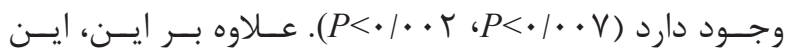

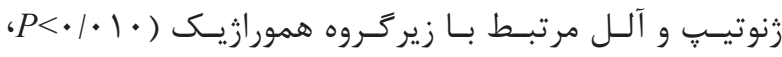

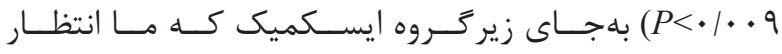

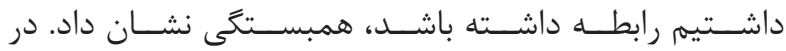

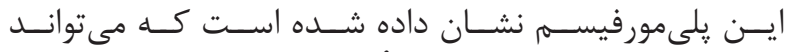

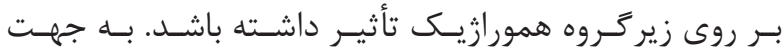


1. Hachiya T, Kamatani Y, Takahashi A, Hata J, Furukawa R, Shiwa Y, et al. Genetic predisposition to ischemic stroke. Stroke. 2017; 48(2): 253-8.

2. Azarpazhooh MR, Etemadi MM, Donnan GA, Mokhber N, Majdi MR, Ghayour-Mobarhan M, et al. Excessive incidence of stroke in Iran. Stroke. 2010; 41(1): e3-e10.

3. Investigators WMPP. The world health organization MONICA project (monitoring trends and determinants in cardiovascular disease): a major international collaboration. J Clin Epidemiol. 1988; 41(2): 105-14.

4. Kumar A, Misra S, Kumar P, Sagar R, Gulati A, Prasad K. Relationship of phosphodiesterase 4D (PDE4D) gene polymorphisms with risk of ischemic stroke: a hospital based case-control study. Neurol Res. 2017; 31: 1-6.

5. Dierick F, Dehas M, Isambert JL, Injeyan S, Bouché AF, Bleyenheuft Y, et al. Hemorrhagic versus ischemic stroke: who can best benefit from blended conventional physiotherapy with robotic-assisted gait therapy? PloS one. 2017 ; 12(6): e0178636.

6. Yuan H, Wang X, Xia Q, Ge P, Wang X, Cao X. Angiotensin converting enzyme (I/D) gene polymorphism contributes to ischemic stroke risk in Caucasian individuals: a meta-analysis based on 22 case-control studies. Int J Neurosci. 2016; 126(6): 488-98.

7. Terni E, Giannini N, Brondi M, Montano V, Bonuccelli U, Mancuso M. Genetics of ischaemic stroke in young adults. BBA Clinical. 2015; 3: 96-106.

8. Sand PG. IL-6 variants in ischemic stroke. Ann Neurosci. 2016; 23(1): 60-1.

9. Tong Y, Wang Z, Geng Y, Liu J, Zhang R, Lin Q, et al. The association of functional polymorphisms of IL-6 gene promoter with ischemic stroke: analysis in two Chinese populations. Biochem Biophys Res Commun. 2010; 391(1): 481-5.

10. Ma Y, Tang R-K, Yang X, Peng G-G, Liu Y, Wang $\mathrm{X}-\mathrm{M}$, et al. Lack of an association between interleukin-6 gene promoter polymorphisms $(-174 \mathrm{G} / \mathrm{C},-572 \mathrm{G} / \mathrm{C})$ and ischemic heart disease and/or ischemic stroke: a metaanalysis. Human Immunology. 2011; 72(8): 641-51.

11. Fishman D, Faulds G, Jeffery R, Mohamed-Ali V, Yudkin JS, Humphries S, et al. The effect of novel polymorphisms in the interleukin-6 (IL-6) gene on IL-6 transcription and plasma IL-6 levels, and an association with systemic-onset juvenile chronic arthritis. J Clin Invest. 1998; 102(7): 1369-76.
منابع

12. Chauhan G, Debette S. Genetic risk factors for ischemic and hemorrhagic stroke. Curr Cardiol Rep. 2016; 18(12): 124. Doi: 10.1007/s11886-016-0804-Z.

13. Pirhoushiaran M, Ghasemi MR, Hami J, Zargari P, Nezhad PS, Azarpazhooh MR, et al. The association of coagulation factor V (Leiden) and factor II (Prothrombin) mutations with stroke. Iran Red Crescent Med J. 2014; 16(11). e11548. Doi: 10.5812/ircmj.

14. Ghasemi MR, Zargari P, Pirhoushiaran M, Azarpazhooh MR, Sadr-Nabavi A. Is apolipoprotein genotype a reason for the excessive incidence of stroke in persian population? J Biomed. 2016; 1(1): 1-6.

15. Zargari P, Ghasemi MR, Pirhoushiaran M, Vakili V, Hami J, Farzadfard MT, et al. Renin-angiotensin a1166c polymorphism and the rrisk of stroke. Journal of Cell and Molecular Research. 2015; 7(1): 32-7.

16. Kumar P, Yadav AK, Kumar A, Sagar R, Pandit AK, Prasad K. Association between Interleukin-6 (G174C and G572C) promoter gene polymorphisms and risk of ischemic stroke: a meta-analysis. Ann Neurosci. 2015; 22(2): 61-9.

17. Becker KJ, Buckwalter M. Stroke, inflammation and the immune response: dawn of a new era. Neurotherapeutics. 2016; 13(4): 659-60.

18. Libby P, Hansson GK. Inflammation and immunity in diseases of the arterial tree. Circ Res. 2015; 116(2): 307-11.

19. Anrather J, Iadecola C. Inflammation and stroke: an overview. Neurotherapeutics. 2016: 13(4): 661-70.

20. Lee JH, Yoon EJ, Seo J, Kavoussi A, Chung YE, Chung SP, et al. Hypothermia inhibits the propagation of acute ischemic injury by inhibiting HMGB1. Molecular Brain. 2016; 9(1): 81. Doi.org/10.1186/s13041-0160260-0.

21. Han D, Liu Z, Wang G, Zhang $Y$, Wu Z. Electroacupuncture improves cognitive deficits through increasing regional cerebral blood flow and alleviating inflammation in CCI rats. Evidence-Based Complementary and Alternative Medicine. 2017. Doi. org/10.1155/2017/5173168.

22. Smith CJ, Emsley HC, Gavin CM, Georgiou RF, Vail A, Barberan EM, et al. Peak plasma interleukin-6 and other peripheral markers of inflammation in the first week of ischaemic stroke correlate with brain infarct volume, stroke severity and long-term outcome. BMC Neurology. 2004; 4(1): 2. Doi.org/10.1186/1471-2377-4-2. 
23. Rallidis LS, Vikelis M, Panagiotakos DB, Rizos I, Zolindaki MG, Kaliva K, et al. Inflammatory markers and in-hospital mortality in acute ischaemic stroke. Atherosclerosis. 2006; 189(1): 193-7.

24. Emsley H, Smith C, Georgiou R, Vail A, Hopkins $\mathrm{S}$, Rothwell N, et al. A randomised phase II study of interleukin-1 receptor antagonist in acute stroke patients. Journal of Neurology, Neurosurgery \& Psychiatry. 2005; 76(10): 1366-72.

25. Licata G, Tuttolomondo A, Di Raimondo D, Corrao S, Di Sciacca R, Pinto A. Immuno-inflammatory activation in acute cardio-embolic strokes in comparison with other subtypes of ischaemic stroke. Thromb Haemost. 2009;101 (5): 929-37.

26. Ota N, Nakajima T, Nakazawa I, Suzuki T, Hosoi T, Orimo $\mathrm{H}$, et al. A nucleotide variant in the promoter region of the interleukin- 6 gene associated with decreased bone mineral density. Journal of Human Genetics. 2001; 46(5): 267-72.

27. Yan J, Greer J, McCombe P. Interleukin 6 promoter $174 \mathrm{G} / \mathrm{C}$ polymorphisms in acute ischemic stroke: $\mathrm{G}$ allele is protective but not associated with IL-6 levels or stroke outcome. Journal of Neuroimmunology. 2016; 293: 22-7. 